\title{
Evaluación de hongos ectomicorrícicos del grupo de los basidiomicetos en la zona del Cofre de Perote, Veracruz
}

\section{Evaluation of ecto-micorrizical fungi from the basidiomicetes group in the zone of Cofre de Perote, Veracruz, Mexico}

\author{
Octavio Córdova-Chávez', Rosario Medel*1, Gerardo Mata², Ranulfo Castillo’ y Jerónimo Vázquez-Ramírez'
}

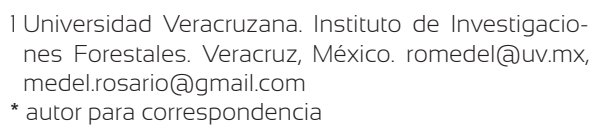

2 Instituto de Ecología, A.C. Red de Manejo Biotecno-

lógico de Recursos. Veracruz, México.

\section{RESUMEN}

La región del Cofre de Perote se encuentra en la parte sur de la Sierra Madre Oriental, en esta zona se encuentra el Parque Nacional Cofre de Perote, el cual presenta altas tasas de deforestación y prácticamente los bosques de oyamel han desaparecido. Esta región alberga gran cantidad de especies fúngicas, algunas muy apreciadas en el mercado local y nacional. La importancia ecológica que desempeñan los hongos en los bosques es más apreciable cuando se toma en cuenta la simbiosis ectomicorrícica. Entre los hongos ectomicorrícicos, los basidiomicetos son más diversos e importantes, dado que entre ellos se encuentran varias especies comestibles. El presente trabajo tiene por objetivo evaluar cuantitativamente especies pertenecientes a este grupo de hongos en seis localidades de la región del Cofre de Perote. La diversidad de hongos ectomicorrícicos encontrados en estas localidades fue de 53 especies de basidiomicetos adscritos a 22 géneros, 15 familias y 6 órdenes. De las especies encontradas 21 resultaron ser comestibles y todas son objeto de venta en el mercado de la ciudad de Xalapa. La diversidad de especies estimada con el índice de Shannon-Wiener osciló entre 1.73 y 3.25. La localidad más diversa para el periodo colectado fue El Conejo (bosque de Abies) y la de menor diversidad fue la Reserva de San Juan del Monte (bosque de Pinus). Las familias más abundantes fueron Amanitaceae, Bankeraceae, Boletaceae y Russulaceae. Las especies encontradas en la mayoría de las localidades fueron Amanita muscaria, Boletus edulis y B. pinophilus.

PALABRAS CLAVE: Agaricales, bosque de pino, diversidad, hongos comestibles, simbiosis.

\section{ABSTRACT}

The Cofre de Perote region is located in the southern part of the Sierra Madre Oriental, located in the area of the Parque Nacional Cofre de Perote which presents high rates of deforestation, and practically the spruce forests are gone. This region is home to a large number of fungal species, some highly valued in the local and national markets. The ecological importance played by fungi in the forests is more appreciable when the ectomicorrhizic symbiosis is taken into account. Among the ectomycorrhizal fungi, Basidiomycetes are more diverse and important given that among them are several edible species, and for this reason, the present work aims to quantitatively assess the species belonging to this group of fungi in six locations in the region of the Cofre de Perote. The diversity of ectomycorrhizal fungi in six locations of the volcano Cofre de Perote, Veracruz, comprised 53 basidiomycete species assigned to 22 genera, 15 families and 6 orders. Twenty-one species were found to be edible and all of them are sold in the market of Xalapa. Species diversity estimated by the Shannon-Wiener index ranged between 1.73 and 3.25. The most diverse locality during the period of the study was El Conejo (Abies forest) and the least diverse was the San Juan del Monte Reserve (Pinus forest). The most abundant families were Amanitaceae, Bankeraceae, Boletaceae, and Russulaceae. The species found in most of the localities were Amanita muscaria, Boletus edulis and B. pinophilus.

KEY WORDS: Agaricales, pine forest, diversity, edible fungi, symbiosis. 


\section{INTRODUCCIÓN}

La región del Cofre de Perote se encuentra en la parte sur de la Sierra Madre Oriental, en esta zona se encuentra el Parque Nacional Cofre de Perote que ocupa 11549 ha y alberga alrededor de 10 comunidades con más de 8000 habitantes (Conanp, 2011). Aunque el Parque Nacional Cofre de Perote es un área protegida, presenta altas tasas de deforestación del orden de 2,3\% anual (Vázquez Torres et al., 2008) y prácticamente los bosques de oyamel han desaparecido (Guzmán, 2013).

Desde el punto de vista micológico, dada su extensión y composición vegetal, esta región alberga gran cantidad de especies fúngicas. Colectas de líquenes realizadas por Humboldt y Bonpland datan de 1804 (Guzmán, 2008). Sin embargo, el primer estudio formal en la zona del Cofre de Perote, es el trabajo de Welden y Guzmán (1978) quienes citaron especies provenientes de varias localidades; posteriormente, Guzmán y Villarreal (1984) citaron 151 especies entre hongos, líquenes y mixomicetos, lo que constituye el inventario más completo de la zona. Trabajos sobre la producción natural de hongos en bosques de la zona del Cofre de Perote fueron realizados por Villarreal y Guzmán (1985, 1986a-b), Villarreal (1987) y Villarreal y Gómez (1997), en los que se aportó importante información sobre la presencia y fenología de especies fúngicas, principalmente comestibles. MontoyaBello et al. (1987) citaron algunas especies de esta zona, particularmente Tricholoma ponderosa (Peck) Sing., especie muy apreciada en el mercado local y nacional. El trabajo etnomicólogico de Jarvis et al. (2004) aportó información sobre los hongos comestibles de una localidad en esta zona y los nombres comunes que reciben; otras aportaciones que han citado especies de esta zona son las de Medel et al. (2006), Guzmán-Olmos (2007) y Medel et al. (2012).

La importancia ecológica que tienen los hongos en los bosques es más apreciada cuando se toma en cuenta la simbiosis ectomicorrícica, que se establece principalmente con más de 32 familias de plantas que incluyen 132 géneros a nivel mundial (Brundrett, 2009). En regiones boreales, templadas y tropicales las micorrizas mantienen el funcionamiento y equilibrio de los mismos (Pérez-Moreno y Read, 2004). Tomando en consideración que entre los hongos ectomicorrícicos, los basidiomicetos son más diversos e importantes, dado que entre ellos se encuentran varias especies comestibles, el presente trabajo tiene por objetivo evaluar cuantitativamente especies pertenecientes a este grupo de hongos en algunas localidades de la región del Cofre de Perote.

\section{MATERIALES Y MÉTODOS}

El área de estudio se localiza en la región NE del Cofre de Perote e incluyó las siguientes localidades: 1) El Conejo (bosque de oyamel, con Abies religiosa como componente principal y en lugares abiertos se puede encontrar Pinus hartwegii y Alnus jorullensis), 2) El Llanillo Redondo (bosque de pino, con Pinus teocote, P. hartwegii, P. montezumae, como componentes principales y en menor medida se encuentran Quercus crassifolia, Alnus jorullensis y Prunus serotina), 3) El Portezuelo (bosque de pino, principalmente Pinus teocote, P. hartwegii, P. montezumae, además de Quercus crassifolia, Alnus jorullensis y Prunus serotina), 4) Los Pescados (ecotono entre bosque de oyamel y bosque de pino, principalmente Abies religiosa, Pinus teocote, P. montezumae, P. hartwegii, y en menor cantidad Cupressus benthamii, Alnus jorullensis, Prunus serotina y Arbutus xalapensis, 5) Reserva de San Juan del Monte (bosque de pino, principalmente Pinus montezumae, P. pseudostrobus, P. teocote, P. patula, con algunos bosquetes de Alnus jorullensis y Quercus crasifolia) y 6) Tembladeras (ecotono de bosque de oyamel y bosque de pino, con Abies religiosa, Pinus montezumae, $P$. hartwegii como componentes principales), en total se muestrearon seis localidades y se elaboró un mapa (Fig. 1) basado en datos de INEGI (2013 a-b) y Conanp (2011). Se recolectaron un total de 145 ejemplares, de julio de 2009 a octubre de 2010. La identificación de los especímenes se realizó siguiendo las técnicas rutinarias en micología (Largent et al., 1977), realizando prepa-

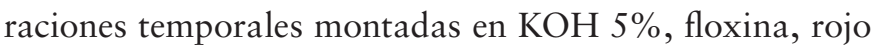
Congo y azul de algodón en lactofenol. El estatus micorrícico se determinó bajo los criterios de Rinaldi et al. (2008), 


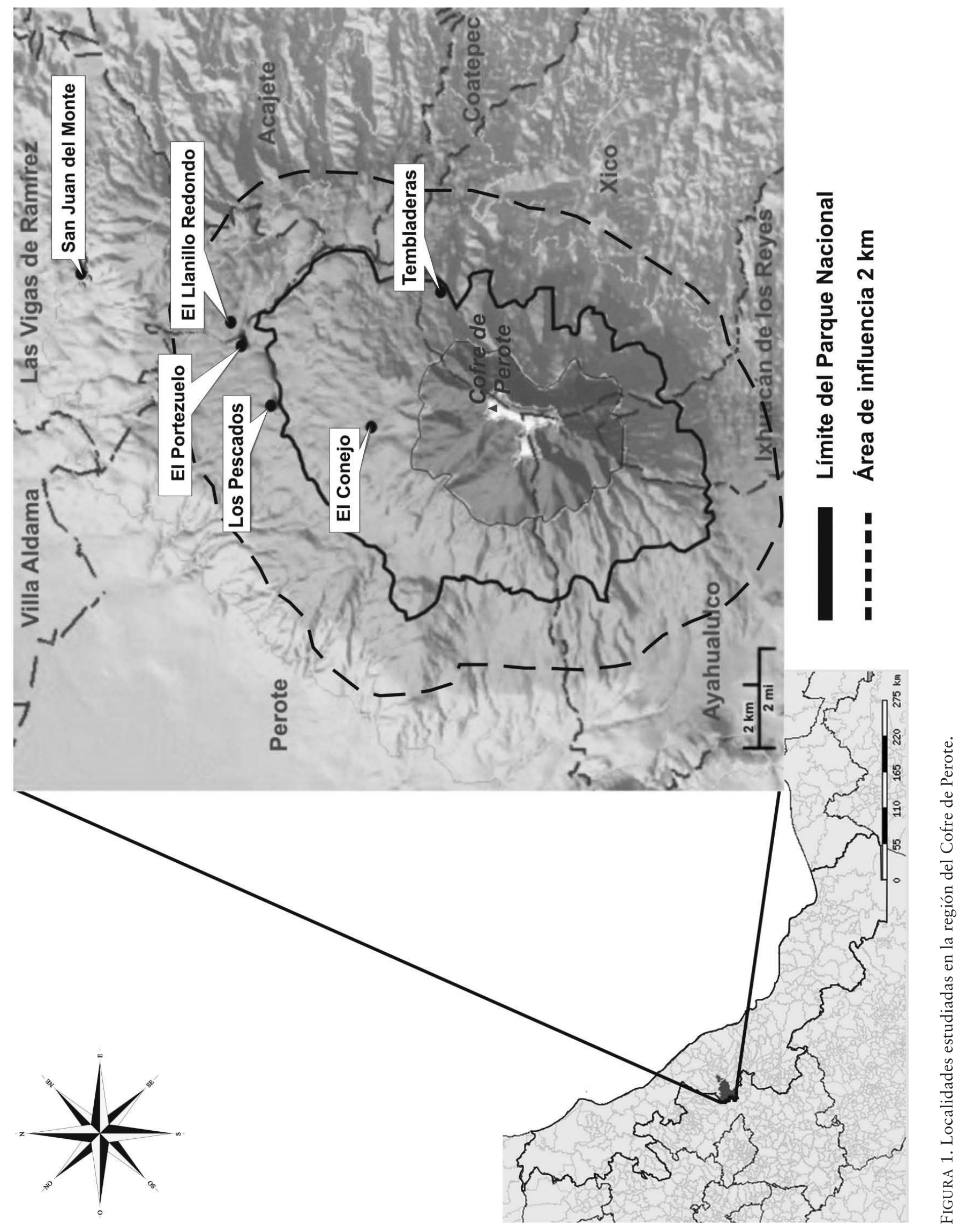


TABla 1. Especies de basidiomicetos ectomicorrícicos en la zona NE del Cofre de Perote.

\begin{tabular}{|c|c|c|c|}
\hline Posición taxonómica & Localidad & Uso & Referencia \\
\hline \multicolumn{4}{|l|}{ AGARICALES, AMANITACEAE } \\
\hline Amanita cokeri (E.-J. Gilbert \& Kühner) E.-J. Gilbert & 2 & & \\
\hline A. muscaria (L.) Lam. & $2 ; 3 ; 4 ; 5$ & & \\
\hline A. pantherina (DC.) Krombh. & $1 ; 5$ & & \\
\hline A. rubescens Pers. & $1 ; 2$ & $C ; M X$ & $2 ; 3$ \\
\hline A. spreta (Peck) Sacc. & 4 & & \\
\hline A. tecomate (Bull.) Lam. & 6 & $\mathrm{C} ; \mathrm{MX}$ & $2 ; 3$ \\
\hline A. vaginata (Bull.) Lam. & 4 & $\mathrm{C} ; \mathrm{MX}$ & \\
\hline \multicolumn{4}{|l|}{ AGARICALES, HYDNANGIACEAE } \\
\hline Laccaria amethystina Cooke & $4 ; 6$ & $\mathrm{C} ; \mathrm{MX}$ & $4 ; 5$ \\
\hline L. laccata (Scop.) Cooke & $2 ; 6$ & $C ; M X$ & $4 ; 5$ \\
\hline \multicolumn{4}{|l|}{ AGARICALES, HYGROPHORACEAE } \\
\hline Hygrophorus chrysodon (Batsch) Fr. & 1 & $C ; M X$ & 4 \\
\hline H. hypothejus (Fr.) Fr. & 1 & & \\
\hline H. nigrescens (Quél.) Quél. & 1 & & \\
\hline \multicolumn{4}{|l|}{ AGARICALES, INOCYBACEAE } \\
\hline Inocybe geophylla (Bull.) P. Kumm. & $1 ; 2 ; 3$ & & \\
\hline \multicolumn{4}{|l|}{ Agaricales, Lyophyllaceae } \\
\hline Lyophyllum decastes (Fr.) Singer & 2 & $\mathrm{C} ; \mathrm{MX}$ & $2 ; 3$ \\
\hline \multicolumn{4}{|l|}{ AGARICALES, TRICHOLOMATACEAE } \\
\hline Tricholoma equestre (L.) P. Kumm. & 6 & & \\
\hline T. imbricatum (Fr.) P. Kumm. & 1 & & \\
\hline T. magnivelare (Peck) Redhead & 4 & $C ; M X ; E$ & $1 ; 2 ; 3$ \\
\hline T. terreum (Schaeff.) P. Kumm. & 1 & & \\
\hline \multicolumn{4}{|l|}{ BOLETALES, BOLETACEAE } \\
\hline Boletus edulis Bull. & $1 ; 2 ; 3 ; 4 ; 5$ & $C ; M X$ & $2 ; 3 ; 5$ \\
\hline B. erythropus Pers. & 6 & & \\
\hline B. pinophilus Pilát \& Dermek & $1 ; 2 ; 3 ; 5$ & $C ; M X$ & $3 ; 5$ \\
\hline B. reticulatus Schaeff. & 4 & $C ; M X$ & 3 \\
\hline Chalciporus piperatus (Bull.) Bataille & 5 & & \\
\hline \multicolumn{4}{|l|}{ BOLETALES, GOMPHIDIACEAE } \\
\hline Chroogomphus vinicolor (Peck) O.K. Mill. & 1 & & \\
\hline \multicolumn{4}{|l|}{ BOLETALES, RHIZOPOGONACEAE } \\
\hline Rhizopogon olivaceoniger A.H. Sm. & 4 & & \\
\hline \multicolumn{4}{|l|}{ BOLETALES, SUILLACEAE } \\
\hline Suillus americanus (Peck) Snell & 1 & $\mathrm{C} ; \mathrm{MX}$ & 4 \\
\hline S. brevipes (Peck) Kuntze & $1 ; 4$ & $\mathrm{C} ; \mathrm{MX}$ & 4 \\
\hline
\end{tabular}


(concluye Tabla 1)

\begin{tabular}{|c|c|c|c|}
\hline Posición taxonómica & Localidad & Uso & Referencia \\
\hline S. granulatus (L.) Roussel & $1 ; 3$ & $C ; M X$ & $4 ; 5$ \\
\hline S. guzmanii G. Moreno, Bandala \& Montoya & 1 & & \\
\hline \multicolumn{4}{|l|}{ CANTHARELLALES, CANTHARELLACEAE } \\
\hline Cantharellus cibarius Fr. & $1 ; 4$ & $C ; M X$ & $2 ; 3 ; 5$ \\
\hline \multicolumn{4}{|l|}{ CANTHARELLALES, HYDNACEAE } \\
\hline Hydnum repandum $\mathrm{L}$. & 1 & & \\
\hline \multicolumn{4}{|l|}{ GOMPHALES, GOMPHACEAE } \\
\hline Gautieria chilensis Zeller \& C.W. Dodge & 1 & & \\
\hline G. mexicana (E. Fisch.) Zeller \& C.W. Dodge & 4 & & \\
\hline Ramaria botrytis (Pers.) Ricken & $1 ; 2$ & $\mathrm{C} ; \mathrm{MX}$ & $2 ; 3$ \\
\hline R. flava (Schaeff.) Quél. & 2 & $\mathrm{C} ; \mathrm{MX}$ & 3 \\
\hline \multicolumn{4}{|l|}{ RUSSULALES, RUSSULACEAE } \\
\hline Lactarius deliciosus (L.) Gray & $2 ; 3 ; 5$ & $\mathrm{C} ; \mathrm{MX}$ & $3 ; 5$ \\
\hline L. mexicanus A. Kong \& Estrada & 1 & & \\
\hline L. rufus (Scop.) Fr. & 1 & & \\
\hline L. salmonicolor R. Heim \& Leclair & $1 ; 4 ; 6$ & $\mathrm{C} ; \mathrm{MX}$ & $4 ; 5$ \\
\hline L. uvidus (Fr.) Fr. & $2 ; 6$ & & \\
\hline Macowanites mexicanus Guzmán & 2 & & \\
\hline Russula alutacea (Fr.) Fr. & 6 & & \\
\hline R. brevipes Peck & 4 & $\mathrm{C} ; \mathrm{MX}$ & $2 ; 3 ; 5$ \\
\hline R. cyanoxantha (Schaeff.) Fr. & $2 ; 4 ; 6$ & & \\
\hline R. delica Fr. & $1 ; 2 ; 3$ & $\mathrm{C} ; \mathrm{MX}$ & 4 \\
\hline R. emetica (Schaeff.) Pers. & 1 & & \\
\hline R. puellaris Fr. & 1 & & \\
\hline \multicolumn{4}{|l|}{ THELEPHORALES, BANIKERACEAE } \\
\hline Bankera fuligineo alba (J.C. Schmidt) Coker \& Beers ex Pouzar & 4 & & \\
\hline Hydnellum suaveolens (Scop.) P. Karst. & 2 & & \\
\hline Sarcodon imbricatus (L.) P. Karst. & $1 ; 2$ & & \\
\hline S. leucopus (Pers.) Maas Geest. \& Nannf. & 4 & & \\
\hline S. scabrosus (Fr.) P. Karst. & 4 & & \\
\hline \multicolumn{4}{|l|}{ TELEPHORALES, THELEPHORACEAE } \\
\hline Thelephora terrestris Ehrh. & $1 ; 4$ & & \\
\hline
\end{tabular}

Notación: Localidades: 1. El Conejo; 2. El Llanillo; 3. El Portezuelo; 4. Los Pescados; 5. San Juan del Monte: 6. Tembladeras. C: Comestibles; E. Exportación; MX: Mercado San José de Xalapa, Veracruz Referencias (en orden alfabético): 1. Bandala et al., 1997; 2. Jarvis et al., 2004; 3. López-Ramírez, 2011; 4. LópezRamírez, com. pers.; 5. Villarreal y Guzmán, 1985 
Tedersoo et al. (2010) y Comandini et al. (2012). Los ejemplares colectados están depositados en la colección micológica del Herbario (XAL) del Instituto de Ecología, A.C., con algunos duplicados en el herbario (XALU) de la Facultad de Biología de la Universidad Veracruzana. Además, se revisaron ejemplares de herbario y la base de datos de hongos de Veracruz de la colección micológica del herbario XAL, todo esto para complementar datos de distribución de las especies, particularmente en la localidad de Tembladeras. Del total de las especies, por cada localidad se obtuvo el índice de diversidad alfa de Shannon-Wiener H', utilzando el programa PAST 3.0 (Hammer et al., 2001). Para lo anterior se elaboró una matriz de datos, calculando la cantidad de especies presentes en cada localidad (riqueza de especies) y la cantidad relativa de recolectas de cada una de las especies expresada como abundancia (Magurran, 1988; Moreno, 2001).

Además, se elaboró una tabla de especies donde se anotaron la localidad, el uso local de las especies, este último dato se basó en los trabajos de Jarvis et al. (2004), López-Ramírez (2011) y se realizaron visitas al mercado San José de Xalapa, donde se venden la mayoría de las especies recolectadas en la zona de estudio (López-Ramírez, com. pers.).

\section{RESULTADOS}

Del estudio e identificación de los 145 hongos recolectados, se encontraron 53 especies exclusivamente ectomicorrícicas. Las especies se adscriben a 22 géneros, 15 familias y 6 órdenes. Las familias más abundantes fueron: Russulaceae con 12 especies, Amanitaceae con siete, Bankeraceae y Boletaceae con cinco, Suillaceae y Gomphaceae con cuatro especies cada una, las demás familias tuvieron una sola especie. De los 22 géneros encontrados, los mejor representados fueron Amanita (siete especies), Russula (seis), Lactarius (cinco), Boletus, Suillus y Tricholoma con cuatro especies cada uno, los demás géneros estuvieron representados con menos de cuatro especies (Tabla 1 ). De los materiales estudiados se encontraron nuevas citas para el estado y el país, los cuales serán tratados en un trabajo taxonómico posterior. Las especies son: Amanita spreta, Gautieria mexicana, Sarcodon leucopus y Rhizopogon olivaceoniger.

De los hongos identificados, $21(39,69 \%)$ resultaron ser comestibles y todos son objeto de venta en el mercado San José de Xalapa (López-Ramírez, 2011), como se anotó en la tabla 1. Llama la atención el caso de Tricholoma magnivelare, especie que era recolectada con fines de exportación (más de 3 t en 1988, según Bandala et al., 1997), pero que recientemente también se ha empezado a ofertar en el mencionado mercado de Xalapa. Otros hongos como Laccaria amethystina y L. laccata se venden mezclados en el mismo mercado (López-Ramírez, com. pers.), Russula delica también es vendida en mercados del centro de México (Pérez-Moreno et al., 2008). Hongos hipogeos como Gautieria mexicana son confundidos con trufas, reciben el nombre de "falsas trufas", no se conoce de su consumo en la zona de estudio o su venta en mercados aledaños, pero sí se ha registrado como comestible en Tlaxcala (Montoya et al., 2004).

La diversidad de especies estimada mediante el índice de Shannon-Wiener osciló entre 1,73-3,25 (Tabla 2), lo que significa una diversidad de baja a media. La localidad más diversa fue El Conejo (bosque de Abies) y la menos la reserva de San Juan del Monte (bosque de Pinus spp.).

\section{DISCUSIÓN}

De los géneros de hongos ectomicorrícicos encontrados, de especial interés son: Boletus llamados "panzas" y muy cotizados por su sabor; las especies de Amanita que resultaron ser tóxicas en su mayoría, a excepción de A. rubescens y A. tecomate que pertenece al complejo de $A$. caesarea llamados "tecomates" en general y que se distinguen entre ellas por caracteres microscópicos (Guzmán y Ramírez-Guillén, 2001) y A. vaginata que es comestible después de la cocción, ya que cruda es tóxica (Pérez-Silva y Herrera, 1991).

Las especies presentes en la mayoría de las localidades fueron Amanita muscaria (en cuatro localidades), Boletus edulis (en cinco) y B. pinophilus (en cuatro).

Se recolectaron especies poco comunes, como Bankera fuligineo alba, Sarcodon leucopus y S. scabrosus, 
cuyas referencias en México son escasas. Con respecto a Gautieria mexicana se encontró que fue citada de Veracruz, sin precisar localidad (Trappe y Guzmán, 1971) por lo que se considera primer registro para la zona de estudio. De la misma manera, Amanita spreta y Rhizopogon olivaceoniger son primeros registros para el estado y en el caso del segundo también lo es para el país.

Respecto a los valores del índice de Shannon-Wiener, en el estudio de Villarreal y Gómez (1997) se obtuvo este índice para localidades cercanas a las de este estudio, encontrándose que, para bosque de Pinus, el índice fue de 4,13 y para el bosque de Pinus-Abies 3,88 , cuando se tomaron el número de cuerpos fructíferos como individuos, ya que estos autores estimaron el mismo índice pero utilizando biomasa (lo cual no se consideró en este estudio, por lo que estos valores usando biomasa no se discuten). Los valores obtenidos en este trabajo fueron más bajos: 2,78-1,73 para bosque de Pinus; 2,78 para Pinus y Abies y 3,25 para bosque de Abies. Aunque los valores del trabajo de Villarreal y Gómez (1997) fueron mayores, no son comparables por la manera en que se realizó la estimación (cuerpos fructíferos), es probable que debido a esto los valores aquí encontrados fueron más bajos, ya que al tomar las recolectas como individuos se dejó fuera cuántos individuos había por colecta.

En los trabajos anteriormente mencionados no se evaluó el bosque de Abies y es precisamente en éste donde se encontró el valor más alto de H’ $(3,25)$ de este estudio, el cual puede estar relacionado con la cantidad de materia orgánica y con una mayor humedad en el mantillo (Pérez-Moreno et al., 2008). Las diferencias entre los valores obtenidos en este estudio se pueden explicar en función de: a) la composición vegetal de las localidades muestreadas, b) el estado de conservación de los sitios, ya que la localidad El Conejo se encuentra dentro del Parque Nacional Cofre de Perote y la Reserva de San Juan está fuera del área de amortiguamiento, a pesar de ser una reserva, lo que se pudo apreciar durante los muestreos,es que esta zona se encuentra deteriorada por deforestación.

Aunque los trabajos realizados en la zona de estudio han mencionado una composición diferente en lo que a las especies se refiere, ésta fue evaluada en localidades distintas a las de este estudio (Villarreal y Guzmán, 1985; Villarreal y Gómez, 1997), sin embargo, algunas de las especies encontradas en este estudio coincidieron en presencia y tipo de vegetación, como se muestra en la tabla 3, estas especies son: Amanita rubescens, A. tecomate (como A. caesarea), Cantharellus cibarius, Lactarius deliciosus, Suillus granulatus y Tricholoma equestre.

Aunque el trabajo de Villarreal y Gómez (1997) versó sobre especies comestibles del Cofre de Perote, estos autores mencionaron 21 especies ectomicorrícicas para bosques de Pinus y 19 para bosques de Pinus-Abies, lo cual contrasta con las 53 especies ectomicorrícicas encontradas en este estudio.

TABLA 2. Valores del Índice de Shannon, riqueza y tipo de vegetación

\begin{tabular}{lccl}
\hline Localidad & $H^{\prime}$ & Riqueza & Vegetación \\
\hline 1. El Conejo & 3,25 & 27 & Bosque de Abies \\
\hline 2. El Llanillo & 2,78 & 17 & Bosque de Pinus spp. \\
\hline 3. El Portezuelo & 1,90 & 7 & Bosque de Pinus spp. \\
\hline 4. Los Pescados & 2,78 & 18 & Ecotono Abies-Pinus \\
\hline 5. San Juan del Monte & 1,73 & 6 & Bosque de Pinus spp. \\
\hline 6. Tembladeras & 2,03 & 9 & Ecotono Abies-Pinus \\
\hline
\end{tabular}


TABLA 3. Especies encontradas en trabajos previos para la misma zona de estudio y su tipo de vegetación.

\begin{tabular}{llll}
\hline Especies & Villarreal y Guzmán, 1985 & Villarreal y Gómez 1997 & $* *$ \\
\hline Amanita rubescens & BP, BPA & BP, BA & BA, BP \\
\hline Amanita tecomate & BP, BPA & BP, BA & BP \\
\hline Cantharellus cibarius & BPA & BP, BA & BA, BPA \\
\hline Lactarius deliciosus & BP, BPA & BP, BA & BP \\
\hline Suillus granulatus & BP, BPA & BP, BA & BP, BA \\
\hline Tricholoma equestre & BP & BA & BP \\
\hline
\end{tabular}

Notacion: BA: bosque de Abies; BP: bosque de Pinus; BPA: Bosque de Pinus-Abies; ** especies citadas en este trabajo.

\section{CONCLUSIONES}

En las seis localidades de este estudio se encontraron un total de 53 especies de basidiomicetos ectomicorrícicos. La diversidad estimada mediante el índice de ShannonWiener, fue de baja a media $(1,73$ - 3,25). De las especies ectomicorrícicas encontradas, aproximadamente 39,6\% (21 especies) fueron comestibles y son vendidas en el mercado local de Xalapa, lo cual coloca a estos hongos ectomicorrícicos como un recurso forestal no maderable importante en la zona del Cofre de Perote. Habitantes de las localidades estudiadas (principalmente El Llanillo Redondo) se dedican a la recolecta de hongos en la época de lluvias y con esta actividad subsisten, siendo para las comunidades un bien importante (Jarvis et al., 2004).

Aunque se ha hablado de la declinación de hongos en diversos bosques, este estudio no tiene por objetivo evaluar este parámetro. Sin embargo, Bandala et al. (1997) mencionaron que en el periodo de 1983-1994, la producción de hongos comestibles en la región del Cofre de Perote disminuyó hasta en $85 \%$, estos datos incluyeron el estudio de Villarreal y Guzmán (1985). Otro estudio de Pérez-Moreno et al. (2010) menciona que la presencia de algunas especies como Amanita caesarea s.l. y Boletus edulis, ha disminuido debido a la destrucción de sus hábitats naturales. Algunos de los factores que han intervenido en esta disminución de especies fueron mencionados por Bandala et al. (1997), como son efectos de la recolecta, factores económicos, tenencia de la tierra, por mencionar algunos.
De acuerdo con García-Romero et al. (2010), la mayor transformación en la superficie forestal del Cofre de Perote se dio entre los años 1960-1970, y a partir de los años 80's la deforestación ha sido en menor grado; estos autores atribuyen los cambios en la superficie forestal, a la dinámica de uso del suelo, concibiéndolo como un problema multifactorial en el que intervienen cuestiones culturales, socioeconómicas y políticas a diversas escalas.

Finalmente, este trabajo es una aproximación para conocer el potencial de los hongos ectomicorrícicos en el Cofre de Perote, recopilar la diversidad de estas especies, constituye la base para llevar a cabo otras investigaciones como son su aislamiento, caracterización y pruebas de micorrización in vitro para poder utilizarlas a nivel de invernadero.

\section{AGRADECIMIENTOS}

El primero de los autores agradece a la DGI-Universidad Veracruzana, la beca otorgada como ayudante de investigación. Al doctor Gastón Guzmán y a los técnicos Florencia Ramírez-Guillén y Juan Lara Carmona el apoyo en la consulta de la colección micológica del herbario XAL y la base de datos de hongos de Veracruz. Al biólogo Armando López Ramírez del INIFOR-UV por la información sobre los hongos comestibles en los mercados de Xalapa. Este trabajo fue financiado por el proyecto FOMIX 108654. 


\section{REFERENCIAS}

Bandala, V.M., L. Montoya e I.H. Chapela. 1997. Wild edible mushrooms in Mexico: A challenge and opportunity for sustainable development. In: M.E.Palm e I.H. Chapela, eds. Mycology in sustainable development: expanding concepts, vanishing borders. Parkway Publishers, Inc. Carolina del Norte. p:76-90.

Brundrett, M.C. 2009. Mycorrhizal associations and other means of nutrition of vascular plants: Understanding the global diversity of host plants by resolving conflicting information and developing reliable means of diagnosis. Plant and Soil 320:37-77.

Comandini, O., A. Rinaldi y T.W. Kuyper. 2012. Measuring and estimating ectomycorrhizal fungal diversity a continuous challenge. In: M. Pagano. Mycorrhiza: occurrence in natural and restored environments. Nova Science Publishers. Nueva York. p:165-200.

Conanp. 2011. Programa de manejo Parque Nacional Cofre de Perote (documento para consulta pública). $135 \mathrm{p}$.

García-Romero, A., Y. Montoya, M.V. Ibarra y G. Graza. 2010. Economía y política en la evolución contemporánea de los usos del suelo y la deforestación en México: el caso del Volcán Cofre de Perote. Interciencia 35:321-328.

Guzmán, G. 2008. Análisis de los estudios sobre los macromycetes de México. Revista Mexicana de Micología 28: 7-15.

Guzmán, G. 2013. Devastación de los bosques y selvas en México. La urgencia de su conservación. Inecol, Xalapa, Veracruz, 68 p.

Guzmán, G. y F. Ramírez-Guillén. 2001. The Amanita caesarea-complex. Bibliotheca Mycologica 187. Cramer, Berlín. $66 \mathrm{p}$.

Guzmán, G. y L. Villarreal. 1984. Estudios sobre los hongos, líquenes y mixomicetos del Cofre de Perote, Veracruz, I. Introducción a la micoflora de la región. Boletín de la Sociedad Mexicana de Micología 19:107-124.

Guzmán-Olmos, R.F. 2007. Macromicetos ectomicorrícicos asociados con Abies religiosa (Kunth) Schltdl. \& Cham. Tesis de licenciatura, Xalapa, Veracruz. Universidad Veracruzana. $80 \mathrm{p}$.

Hammer. Ø., D.A.T. Harper y P.D Ryan. 2001. PAST: Paleontological statistics software package for education and data analysis. Paleontología Electrónica 4(1): 9 pp. http:// palaeo-electronica.org/2001_1/past/issue1_01.htm.

INEGI. 2013a.http://sc.inegi.org.mx/sistemas/cobdem/consultapor-ageo.jsp? recargar=true consultada en octubre 2013.

INEGI. 2013b. http://gaia.inegi.org.mx/mdm5/viewer.html, consultada en octubre 2013.

Jarvis, M.C., A. M. Miller, J. Sheahan, K. Ploetz, J. Ploetz, R. Watson, M. Palma, C.A. Pascasio, J. García, A. López y B. Orr. 2004. Edible wild mushrooms of the Cofre de Perote region, Veracruz, Mexico: An ethnomycological study of common names and uses. Economic Botany 58:111-115.

Largent, D., D. Johnson y R. Watling. 1977. How to identify Mushrooms to Genus III: Microscopic features. Mad River Press Incorporate. Eureka, California. 148 p.

López-Ramírez, M.A. 2011. Los hongos: recurso natural forestal y su aprovechamiento sustentable. Editorial Académica Española. 108 p.

Magurran, A.E. 1988. Ecological diversity and its measurement. Chapman and Hall. Londres, 179 p.

Medel, R., F.D. Calonge y G. Guzmán. 2006. Nuevos registros de Pezizales (Ascomycota) de Veracruz. Revista Mexicana de Micología 23:83-86.

Medel, R., Y. Baeza, G. Mata y D. Trejo. 2012. Ascomicetos ectomicorrízicos del Parque Nacional Cofre de Perote, Veracruz, México. Revista Mexicana de Micología $35: 43-47$.

Montoya, A., A. Kong, A. Estrada-Torres, J. Cifuentes y J. Caballero. 2004. Useful wild fungi of La Malinche Nacional Park, Mexico. Fungal Diversity 17:115-143.

Montoya-Bello, L., V.M. Bandala y G. Guzmán. 1987. Nuevos registros de hongos del estado de Veracruz IV. Revista Mexicana de Micología 3:83-107.

Moreno, C. 2001. Métodos para medir la biodiversidad. Vol. 1. Programa Iberoamericano de Ciencia y Tecnología para el Desarrollo, Oficina Regional de Ciencia y Tecnología para América Latina y el Caribe de UNESCO y Sociedad Entomológica Aragonesa. Serie Manuales y Tesis SEA. 84 p.

Pérez-Moreno, J. y D.J. Read. 2004. Los hongos ectomicorrízicos, lazos vivientes que conectan y nutren a los árboles en la naturaleza. Interciencia 29(5):239-247. 
Pérez-Moreno, J., M. Martínez-Reyes, A. Yescas-Pérez, A. Delgado Alvarado y B. Xoconostle-Cázares. 2008. Wild mushroom markets in central Mexico and a case study at Ozumba. Economic Botany 62(3):425-436.

Pérez-Moreno, J., A. Lorenzana, F.V. Carrasco-Hernández y A. Yescas-Pérez. 2010. Los hongos comestibles silvestres del Parque Nacional Izta-Popo. Zoquiapan y Anexos. Colegio de Posgraduados, Semarnat, Conacyt. Montecillo, Texcoco, Edo. de México. 167 p.

Pérez-Silva, E. y T. Herrera. 1991. Iconografía de macromicetos de México I Amanita. Instituto de Biología. Publicaciones Especiales 6. UNAM. 136 p.

Rinaldi, A.C., O. Comandini, y T.W. Kuyper. 2008. Ectomycorrhizal fungal diversity: separating the wheat from the chaff. Fungal Diversity 33:1-45.

Tedersoo, L., T.W. May y M.E. Smith. 2010. Ectomycorrhizal lifestyle in fungi: global diversity, distribution, and evolution of phylogenetic lineages. Mycorrhiza 20:217-263.

Trappe, J.M., y G. Guzmán. 1971. Notes on Some hypogeous Fungi from Mexico. Mycologia. 63(2):317-332.

Vázquez-Torres, S.M, C.I. Carvajal-Hernández y A.M. AquinoZapata. 2008. Áreas Naturales Protegidas. Conanp. Consultado el 4 de marzo, 2013. Disponible en:http://cdigital.uv.mx/ bitstream/123456789/9656/1/10ANPSVERDEOSC.pdf.

Villarreal, L. y G. Guzmán. 1985. Producción de los hongos comestibles silvestres en los bosques de México (Parte I). Revista Mexicana de Micología 1:51-90.
Villarreal, L. y G. Guzmán. 1986a. Producción de los hongos comestibles silvestres en los bosques de México (Parte III). Revista Mexicana de Micología 2:259-277.

Villarreal, L. y G. Guzmán. 1986b. Producción de los hongos comestibles silvestres en los bosques de México II. Biotica $11: 271-280$.

Villarreal, L. 1987. Producción de los hongos comestibles silvestres en los bosques de México (Parte IV). Revista Mexicana de Micología 3:265-282.

Villarreal, L. y A. Gómez. 1997. Wild edible mushrooms in Mexico: A challenge and opportunity for sustainable development. In: M.E. Palm e I.H. Chapela, eds. Mycology in sustainable development: expanding concepts, vanishing borders. Parkway Publishers, Inc. Carolina del Norte. p:99-108.

Welden, L. y G. Guzmán. 1978. Lista preliminar de los hongos, líquenes y mixomicetos de las regiones de Uxpanapa, Coatzacoalcos, Los Tuxtlas, Papaloapan y Xalapa (parte de los estados de Veracruz y Oaxaca). Boletín de la Sociedad Mexicana de Micología 12:59-102.

Manuscrito recibido el 6 de marzo de 2013

Aceptado el 10 de diciembre de 2013.

Este documento se debe citar como:

Córdova-Chávez, O., R. Medel, G. Mata, R. Castillo y J. Vázquez-Ramírez. 2014. Evaluación de hongos ectomicorrícicos del grupo de los basidiomicetos en la zona NE del Cofre de Perote, Veracruz. Madera y Bosques 20(1):97-106. 